\title{
Cyclic Diamondoid Structures with Shared Vertices, Edges, or 6-membered Rings
}

\author{
Alexandru T. Balaban \\ Texas A\&M University Galveston, 200 Seawolf ParkwayGalveston, \\ TX 77553-1675, USA
}

RECEIVED APRIL 18, 2014; REVISED OCTOBER 11, 2014; ACCEPTED NOVEMBER 7, 2014

\begin{abstract}
Diamondoid structures with shared vertices, edges, or 6-membered rings can theoretically be curved into toroidal structures whose calculated energy provides information about steric strain. Diamondoid hydrocarbons sharing one vertex between two adamantane units are called $[n]$ spiromantanes, where $n$ indicates the number of adamantane units. When a pair of adamantane units shares one CC bond, the resulting assembly is called one-edge- $[n]$ mantane, specifying (by letters in square brackets) which bonds are shared by the adamantane units. Two adjacent edges may be shared by a pair of adamantane units, and the assembly is called two-edge- $[n]$ mantane, again specifying by letters in square brackets the shared bonds. Catamantanes or perimantanes sharing a 6-membered ring of carbon atoms may form larger rings in an assembly which is called $[n]$ cyclomantane; in the case of catamantanes, the structure of the diamondoid is specified by codes of the dualists. Finally, nanotubes derived from hexagonal diamond, as well as corresponding toroidal structures, are discussed.
\end{abstract}

Keywords: diamondoids, cyclic structures, spiromantanes

\section{INTRODUCTION}

Diamond hydrocarbons (called diamondoids for brevity) have obtained prominence since their discovery in petroleum. ${ }^{1-3}$ They contain adamantane units sharing chair-shaped hexagons of carbon atoms. Their isolation and purification are based on their high stability towards heat and oxidation. Schleyer and coworkers discovered that adamantane $\left(\mathrm{C}_{10} \mathrm{H}_{16}\right),{ }^{4}$ diamantane $\left(\mathrm{C}_{14} \mathrm{H}_{20}\right){ }^{5}$ and triamantane $\left(\mathrm{C}_{18} \mathrm{H}_{24}\right)^{6}$ can be obtained synthetically by Lewis-acid or transition-metal catalyzed rearrangements of polycyclic isomers; the driving force in such reactions is based on the fact that diamondoids have appreciably lower steric strain in comparison with all other polycyclic isomers. However, only one of the three isomeric tetramantanes could be obtained by this approach, ${ }^{7}$ so that for higher diamondoids the only source at present is the isolation from petroleum. Among tetramantane isomers, one is chiral (2 enantiomers) so that there are actually four possible tetramantanes $\mathrm{C}_{22} \mathrm{H}_{28}{ }^{8}$ Several reviews on diamondoids have been published. ${ }^{9-14}$

A similarity with benzenoid hydrocarbons exist, because benzenoids are also formed from units (sixmembered aromatic rings) sharing $\mathrm{CC}$ bonds. Balaban and Harary had devised for benzenoids a classification relying on dualists; ${ }^{15}$ they consist of vertices in the center of each unit and of edges connecting vertices in adjacent rings. The 2D honeycomb lattice and the trigonal lattice are reciprocal dualist lattices. Then the classification includes catafusenes that are benzenoids with acyclic dualists, perifusenes that are benzenoids with dualists having 3-membered rings, and coronafusenes that are benzenoids with dualists having larger rings that are not peripheries of 3-membered ring aggregates. For catafusenes, a code was devised based on digits 1,2 , and 3 for the three directions around $\mathrm{sp}^{2}$ hybridized carbon atoms. ${ }^{15,16}$

For specifying constitutional structures of diamondoids, Balaban and Schleyer conceived a similar system that is also based on their dualists. ${ }^{17}$ In this case, the 3D diamond lattice is self-dual. A diamondoid formed from $n$ adamantane units with an acyclic dualist is called an $[n]$ catamantane, but catamantanes are of two kinds: regular catamantanes when the molecular formula is $\mathrm{C}_{4 n+6} \mathrm{H}_{4 n+12}$ and irregular ones when there are fewer $\mathrm{C}$ and $\mathrm{H}$ atoms for a given $n$ value. Perimantanes have dualists with 6-membered rings, and coronamantanes have dualists with larger rings that are not perimeters of 6-membered ring aggregates. For constitutional isomers

\footnotetext{
$\dagger$ Dedicated to Professor Mirjana Eckert Maksić on the occasion of her $70^{\text {th }}$ birthday.

* Author to whom correspondence should be addressed. (E-mail: balabana@tamug.edu)
} 


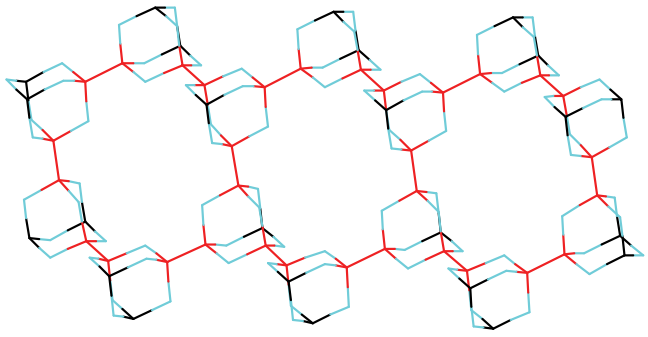

Figure 1. A necklace-shaped structure with three cycles, each of which has six adamantane units.

of catamantanes, a code was proposed based on digits 1 to 4 indicating the four directions around $\mathrm{sp}^{3}$-hybridized carbon atoms. ${ }^{17-19}$

Tertiary carbon atoms in diamondoid hydrocarbons usually undergo substitution more easily than secondary carbon atoms, and this property was intensely used for converting diamondoids from petroleum into a wide variety of compounds. ${ }^{20-27}$

In the following we will discuss diamondoid structures that share among neighboring adamantane units: one vertex, one edge, two adjacent edges, or 6membered rings (the latter diamondoids may be catamantanes or perimantanes). Special attention will be paid to such assemblies of adamantane units that are cyclic, forming larger toroidal structures. Synthetic approaches will not be discussed, since they need to be diversified for the various classes of cyclodiamondoids. Polymantanes are considered here to be diamondoids whose dualists are subgraphs of the diamond lattice.

Nanotubes (single-walled abbreviated as SWNTs, or multi-walled abbreviated as MWNTs) are carbon allotropes with various dispersion values, i.e. mixtures from a chemical viewpoint. By contrast, diamond hydrocarbons, called diamondoids for brevity, which are isolated and purified from petroleum, are substances. Both nanotubes and diamondoids can be functionalized by attaching to them various substituents or functional groups. For physical, chemical, or biological applications, unitary substances should be preferred over homologous mixtures, if costs are similar. Cyclic toroidal SWNTs are known. In the following, various cyclic assemblies of "spiranic necklaces" of diamondoids or of toroidal diamondoids are reviewed; their calculated
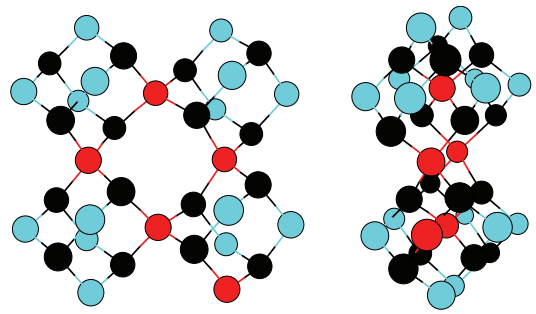

Figure 2. Low-strain cyclo[4]spiromantane involving four shared proximal $\mathrm{CH}_{2}$ groups which become quaternary carbon atoms (front and side view).

energies (to be published in future papers) can provide indications about steric strain as function of the number of adamantane units involved in the cyclic structure.

\section{Necklace-Shaped Aggregates of Isolated Adaman- tane Units}

Each of the six secondary $\mathrm{CH}_{2}$ groups of adamantane has four "proximal $\mathrm{CH}_{2}$ groups" and one "opposite $\mathrm{CH}_{2}$ group". Trivial diamondoids with isolated adamantane units, i.e. necklace-shaped structures, will not be discussed in detail. There can be little extra steric strain in some systems that have bonds involving tertiary carbon atoms of adamantane with one or several cycles; also for carbon atoms originating in proximal $\mathrm{CH}_{2}$ groups (as seen for the structure with three large rings in Figure 1 with each adamantane unit mimicking a carbon atom in per-hydrogenated anthracene) or a minimal steric strain for structures with mixed bonds between carbons from $\mathrm{CH}_{2}$ and $\mathrm{CH}$ groups. In all figures, hydrogen-depleted structures will be shown. In all such structures the adamantane units can rotate around the bonds between adamantane units.

\section{Diamondoid Structures Sharing One Carbon Atom for Two Adamantane Units ([n]spiromantanes)}

Two adamantane units sharing one carbon atom from their secondary carbon atoms form a spiranic structure. For brevity, we will call such diamondoids ' $[n]$ spiromantanes', with $n$ in square brackets denoting the number of adamantane units. The hydrocarbon [2] spiromantane $\mathrm{C}_{19} \mathrm{H}_{28}$ was synthesized by two routes, ${ }^{28,29}$ and thermal rearrangements of 1-substituted derivatives
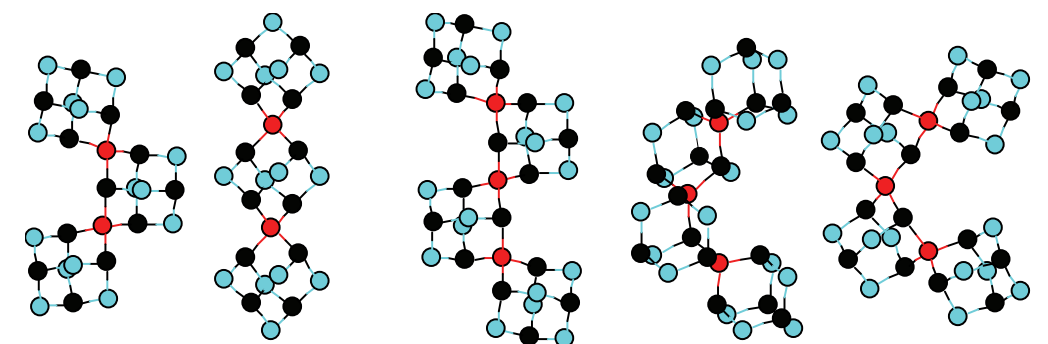

Figure 3. Open nonbranched chains of $[n]$ spiromantanes: two for $n=3$, involving proximal or distal $\mathrm{CH}_{2}$ groups (which become quaternary carbon atoms) and three for $n=4$. 


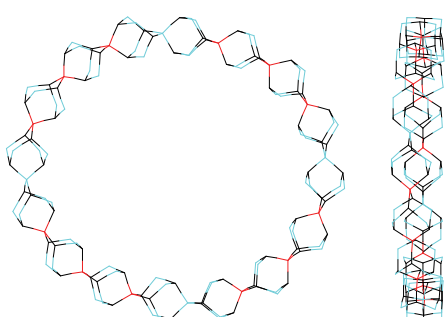

Figure 4. Cyclo[16]spiromantane (front and side view) involving nonbranched distal $\mathrm{CH}_{2}$ group.

were reported. ${ }^{30,31}$ For certain numbers $n$ of adamantane units (Figure 2 for $n=4$ ), cyclic low-strain assemblies are possible (cyclo[4]spiromantane) with spiranic carbon atom bridges involving proximal $\mathrm{CH}_{2}$ groups of adamantane units. The following color code will be common to all figures in the present communication: red for quaternary carbon atoms, black for tertiary $(\mathrm{CH})$ groups and blue for secondary $\left(\mathrm{CH}_{2}\right)$ groups.

There are two possibilities for an open chain of spiromantanes involving three adamantane units involving either proximal or distal $\mathrm{CH}_{2}$ groups. For spiromantanes with four adamantane units, open chains may be either non-branched with three such possibilities (Figure 3) or branched: for four adamantane units, $\mathrm{CH}_{2}$ groups may be all proximal, or one pair is distal. With larger $n$ values, the number of possibilities increases combinatorially. The branched spiro[7]mantane with six octahedrally-situated branches is more strained.

Open chains of spiranic structures have little strain, but macrocyclic spiranic structures involving carbons of distal $\mathrm{CH}_{2}$ groups in adamantane units with various $\mathrm{n}$ values that form a ring are strained. Figure 4 presents a front and side view of cyclo[16]spiromantane derived from nonbranched distal $\mathrm{CH}_{2}$ groups.

Although calculations for strain energies will be reported separately, at present one can report that torsion and 1,4-van der Waals interactions are the main contributors (with minor contributions from stretch and bend). "Open" distal $[n]$ spiromantanes have linearly increasing strain energies with increasing $n$, but cyclic ones with low $n$ values have also an antagonistic bond angle effect that decreases exponentially with increasing $n$, so that the total strain energy is minimal at $n=10$. Similar trends are also found for diamondoids sharing edges or hexagonal faces of adamantane units.
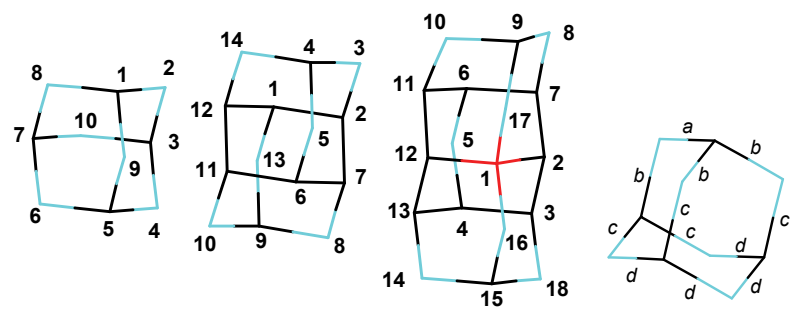

Figure 5. Numbering of carbon atoms in adamantane, diamantane, and triamantane; edge labelings in adamantane.

\section{Nomenclature of Diamondoids}

The Balaban-Schleyer codes specify constitutional isomerism, but for stereoisomerism the IUPAC-von Baeyer code must be employed. The IUPAC numbering of carbon atoms in the first three diamondoids (adamantane, diamantane which initially was called congressane, and triamantane that have just one isomer each) is shown in Figure 5.

Adamantane is tricyclo[3.3.1.1 $\left.1^{3,7}\right]$ decane. It has molecular formula $\mathrm{C}_{10} \mathrm{H}_{16}$ and partitioned formula according to the types of carbon atoms $(\mathrm{CH})_{4}\left(\mathrm{CH}_{2}\right)_{6}$.

Diamantane is pentacyclo[7.3.1.1 $\left.1^{4,12} \cdot 0^{2,7} \cdot 0^{6,11}\right]$ tetradecane and has code [1]. It has molecular formula $\mathrm{C}_{14} \mathrm{H}_{20}$ and partitioned formula $(\mathrm{CH})_{8}\left(\mathrm{CH}_{2}\right)_{6}$.

Triamantane is heptacyclo[7.7.1.1 $1^{3,15} \cdot 0^{1,12} \cdot 0^{2,7}$. $0^{4,13} \cdot 0^{6,11}$ ]octadecane and has code [12]. It has molecular formula $\mathrm{C}_{18} \mathrm{H}_{24}$ and partitioned formula $\mathrm{C}(\mathrm{CH})_{10}\left(\mathrm{CH}_{2}\right)_{7}$.

Just as in the more familiar case of benzenoids, edges will be designated by italic letters in square brackets. Figure 5 also shows the labeling of adamantane edges relatively to one another: if one CC bond is designated as $a$, there are three more types of bonds designated as $b, c$, and $d$. For adamantane units sharing one bond, there are therefore three possibilities, to be examined in the next section, namely $a c$, and two kinds of $a d$ bond sharing, because there are two different types of $d$ bonds relatively to the $a$ bond: being or not being edges of the same hexagon.

An adamantane unit may share three or four (but not more) edges, each with another adamantane unit. Since for large-ring monocyclic assemblies such sharing is not relevant, it will not be discussed here.

\section{Diamondoid Structures Sharing One C-C Bond for Two Adamantane Units (One-edge- $[n]$ mantanes)}

As mentioned in the preceding section, there are three possibilities for sharing one $\mathrm{C}-\mathrm{C}$ bond for two adamantane units. Taking as example

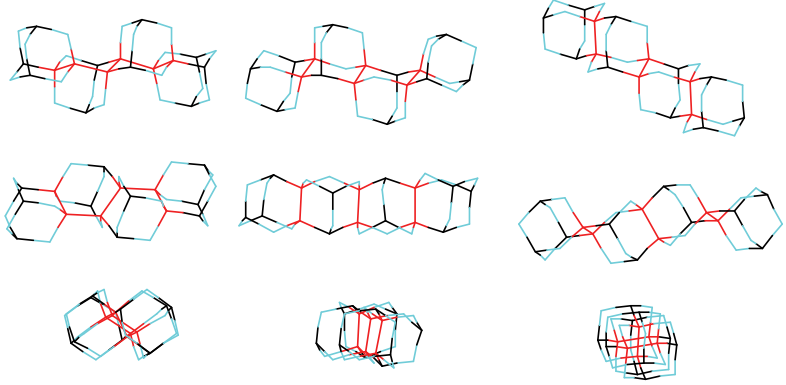

Figure 6. Three columns for the three different open-chain assemblies with four adamantane units sharing one edge (side view on $1^{\text {st }}$ row, front view on $2^{\text {nd }}$ row, and view along long axis on $3^{\text {rd }}$ row. From left to right, one-edge-[4,a,c]mantane, one-edge- $[4, a, d]$ mantane- $0^{\circ}$ (parallel bonds), and one-edge$[4, a, d]$ mantane- $90^{\circ}$ (orthogonal bonds). 


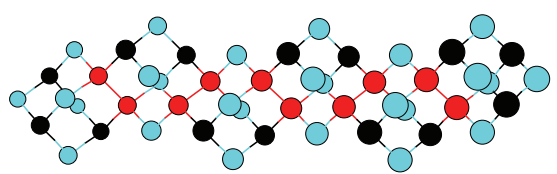

Figure 7. Low-strain open-chain assembly for one-edge$[6, a, d]$ mantane- $90^{\circ}$.

one-edge-[4]mantanes, Figure 6 presents these possibilities. For the first one, it can be seen in the side view that adamantane units alternate up and down on a zigzag perhydrophene quasi-flat long stretch of hexagons with shared bonds at angles $120^{\circ}$ (one-edge-[4a,c]mantane); for the next (one-edge-[4a,d]mantane) $-0^{\circ}$ the adamantne units again alternate up and down but on a linear perhydroacene quasi-flat long stretch of hexagons with parallel shared bonds; and for the last (one-edge$[4 a, d]$ mantane $)-90^{\circ}$ the adamantane units have orthogonal shared bonds. All these open-chain assemblies have low steric strain as indicated above, and Figure 7 shows such examples in ball-and-stick representation.

Cyclic assemblies have low steric strain for oneedge-cyclo $[n, a, d]$ mantane- $0^{\circ}$ with certain numbers $n$ of adamantane units (Figure 8 for $n=6$ ) or higher strained for any $n \neq 6$ values (Figure 9).

A different type of one-edge-sharing occurs when the bonds involved are $a c$. In such a case cyclic assemblies are strained for any $n$ values (Figure 10).

\section{Diamondoid Structures Sharing Two Adjacent C-C Bonds for Two Adamantane Units (Two-edge- [n]mantanes)}

When two adjacent edges $\mathrm{CH}-\mathrm{CH}_{2}-\mathrm{CH}$ (tertiarysecondary-tertiary or $t-s-t$ ) are shared by a pair of adamantane units, there is a slight curvature of this pair, as seen in Figure 11, because when all units have the same orientation, with a sequence of quaternary carbon atoms alternating with secondary $\mathrm{CH}_{2}$ groups, there is steric repulsion between hydrogens of the $\mathrm{CH}_{2}$ groups. If a higher curvature is imposed when a cyclic assembly is created as displayed by Figure 12, steric strain results. Names now involve italic letters in sequences of edges for middle adamantane units. Assemblies sharing edge pairs between two adamantane units that involve $\mathrm{CH}_{2}-\mathrm{CH}-\mathrm{CH}_{2}$ bonds are not possible.
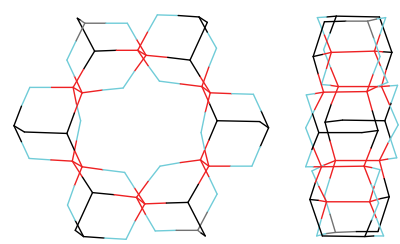

Figure 8. Low-strain macrocyclic assembly for one-edgecyclo $[6, a, d]$ mantane $-0^{\circ}$.

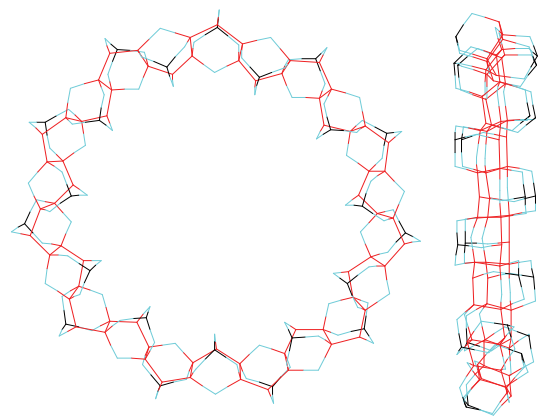

Figure 9. Front and side views of strained macrocyclic oneedge-cyclo[22,a,d]mantane- $0^{\circ}$.
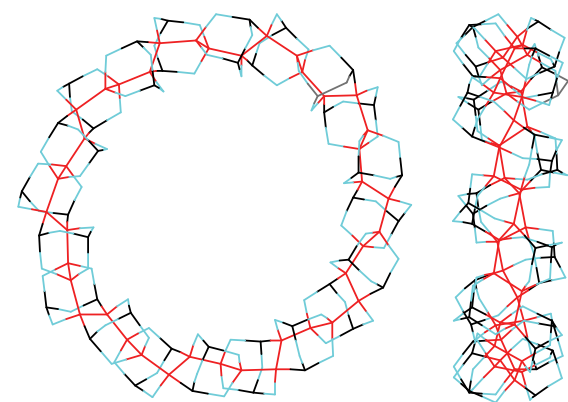

Figure 10. A one-edge-sharing cyclo[20,a,c]mantane (front and side view).

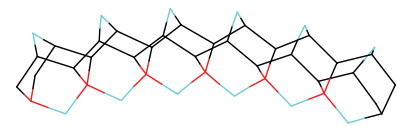

Figure 11. Acyclic assembly of six adamantane units sharing $t-s-t$ pairs of edges $[6, a b, e f]$ mantane.
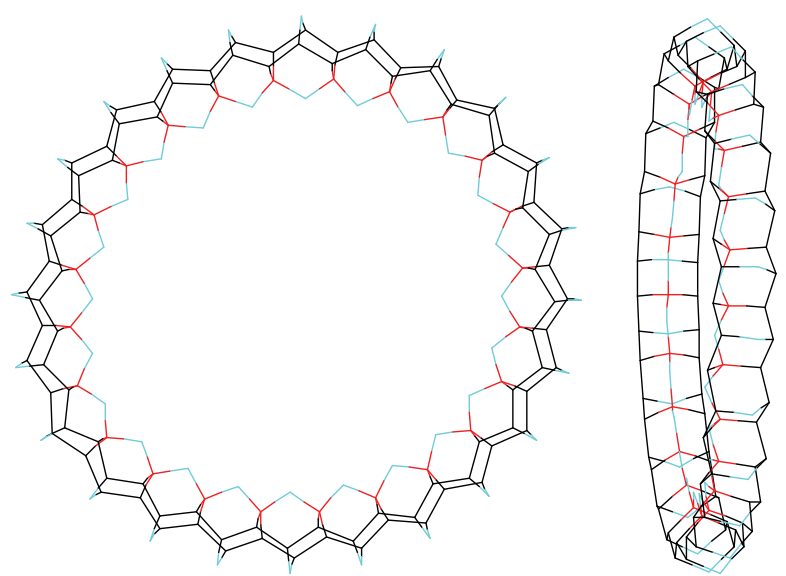

Figure 12. Front and side views for a cyclic assembly sharing two adjacent $\mathrm{C}-\mathrm{C}$ bonds for each pair of adamantane units in cyclo[24,ab,ef]mantane. 

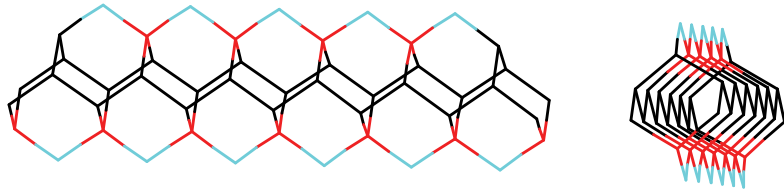

Figure 13. Two views of a short zigzag catafusene for comparing with Figure 11.

In Fig. 13 on can see that if the upper secondary groups from Fig. 11 become connected, the steric repulsion between hydrogen atoms vanishes and there is no longer any curvature. Now the assembly is a catafusene that shares hexagons, as will be discussed in the next section.

\section{Catafusene Structures Sharing One 6-membered Ring for Two Adamantane Units}

For better visualization, we start by showing in Figure 14 an open-chain assembly of benzenoid rings sharing one chair-shaped hexagon of carbon atoms for each adjacent pair of adamantane units. The dualist is indicated by a chain of letters $C$ for each adamantane unit. The catafusene has a blade-like structure, with the dualist code 12121212121212121212121212121. Such zigzag catafusenes may be useful as raw materials for affording substituted compounds with biological activity due to interaction with enzyme receptors at precisely defined geometries. Based on the POLCYC computer program, ${ }^{32}$ the IUPAC von Bayer nomenclature and carbon numbering were devised for all such zigzag catafusenes. $^{33}$

If the ends of an assembly similar to Figure 14 are connected into a cyclic structure, and if the dualist is now represented by olive-colored balls, the result is shown on the left part of Figure 15 as [26]cyclomantane in a ball-and-stick representation. On the right-hand side and without the dualist, the same [26]cyclomantane is shown in a representation with sticks for $\mathrm{C}-\mathrm{C}$ bonds. The large-ring structures no longer fit the molecular formulas defining catamantanes.

It is evident from Figure 16 that the $\mathrm{C}-\mathrm{C}$ bond lengths cannot be equal. The larger the ring, the lower is the strain. Assemblies with less than 26 adamantane

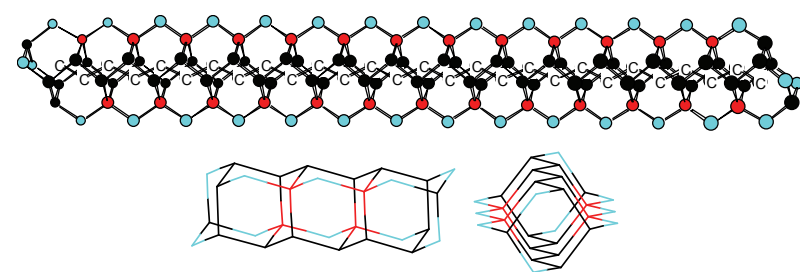

Figure 14. An acyclic zigzag assembly of 28 adamantane units sharing six-membered rings (the dualist is represented by a sequence of letters $\mathrm{C}$ in the Centers of adamantane units); then zigzag pentamantane is shown viewed sideways and along the long axis.
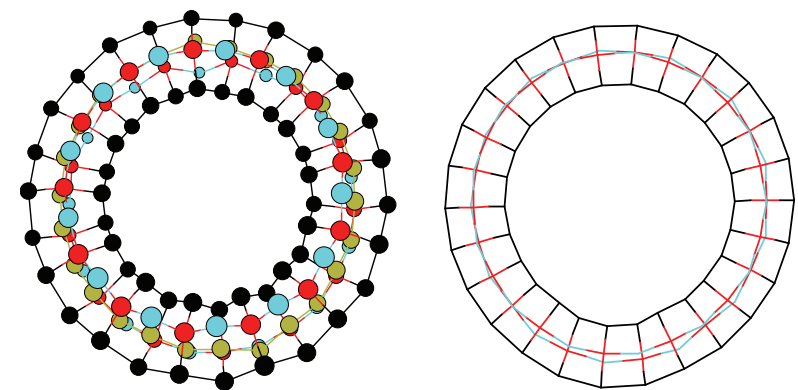

Figure 15. Left: [26]cyclomantane with dualist, in ball and stick representation; right: the same, without dualist, in stick representation.

units have no longer flat rings and 'smooth' dualists as those in Figure 15.

\section{Large-ring Perifusene Structures}

As examples for perifusenes, we selected diamondoids whose dualists are all-trans-perhydroacenes; such blade-shaped structures have IUPAC von Baeyer names and numberings of carbon atoms were discussed in an earlier paper. ${ }^{34}$ Figure 16 presents such a perifusene whose dualist is isomorphic with 2,7-dimethyl-all-transperhydroanthracene.

Considerable strain is associated with cyclic structures based on such perimantane systems, illustrated by Figure 17.

In addition to perimantanes belonging to the preceding type, one can also have perimantanes whose dualists are all-cis-perhydroacenes. For better view, the perhydrohexacenes in Figure 18 are displayed as hydrocarbons that include hydrogen atoms represented by blue dangling bonds.

In Fig. 19 one can see a macrocyclic assembly of adamantane units resulting in a perimantane whose dualist is an all-cis-perhydroacene. Unlike the systems with all-trans-perhydrohexacene dualists that appear in side views like acenes, in the present case the side view appears like zigzag phenes.

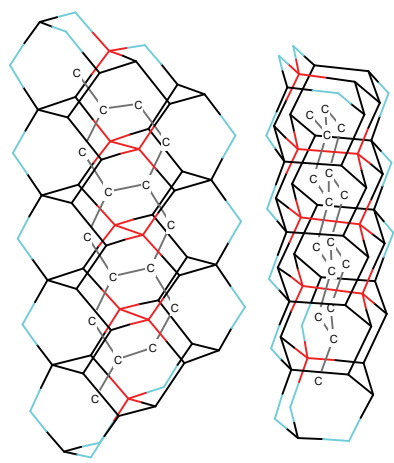

Figure 16. Front and side views of the perifusene having a dualist that is isomorphic with 2,7-dimethyl-all-transperhydroanthracene (with carbon skeleton composed of letters C). 

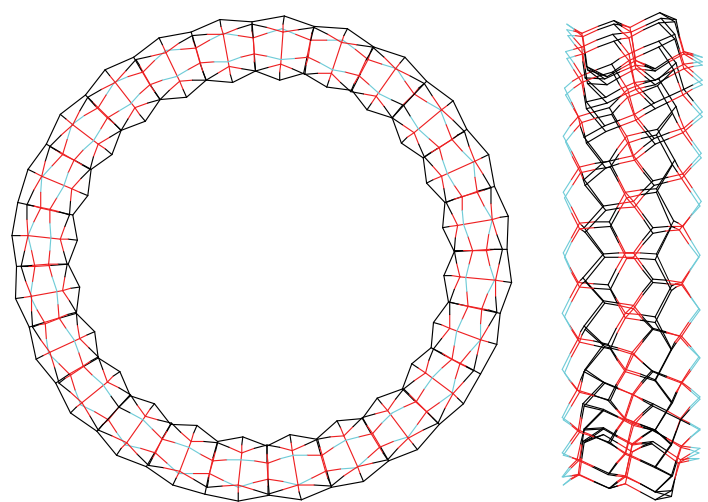

Figure 17. Front and side views of a cyclic perimantane assembly.

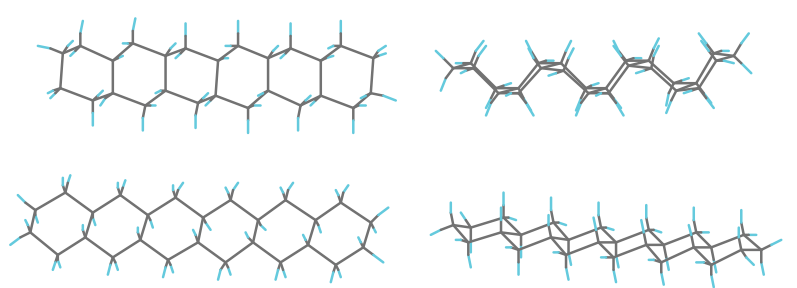

Figure 18. Upper row: front and side view of all-cisperhydrohexacene dualist for an open-chain of perimantane; lower row: same for all-trans-perhydrohexacene dualist.
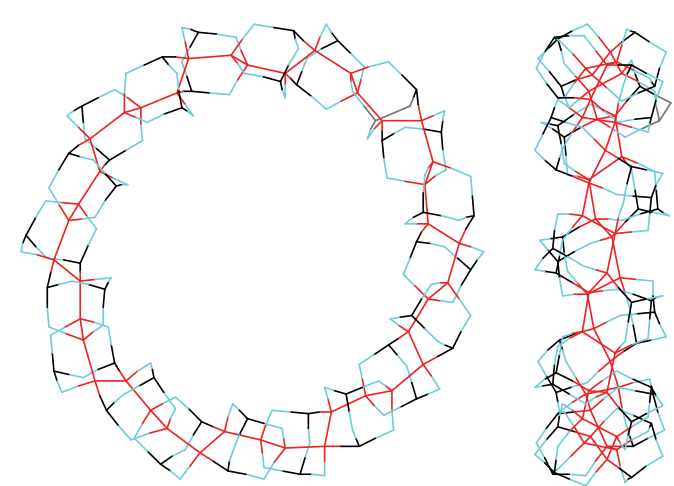

Figure 19. Front and side views of a macrocyclic assembly for a perimantane whose dualist is an all-cis-perhydroacene.
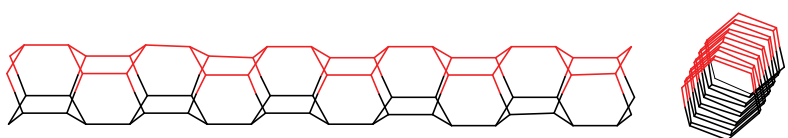

Figure 20. Nanotube from the lonsdaleite lattice: side-view and view along the axis of the nanotube. Here and in the next Figure the color serves only to facilitate viewing one side of the structure.

\section{Nanotubes and Corresponding Tori Derived from Hexagonal Diamond (Londaleite)}

A different form of diamond, having eclipsed bonds, was discovered in 1967 and it is called lonsdaleite. Its crystalline lattice is isomorphic with that of wurtzite (one form of zinc sulfide), whereas another crystalline form of $\mathrm{ZnS}$ (sphalerite) is similar to the diamond lattice that has only staggered bonds. Puckered honeycomb carbon layers stacked in $\mathrm{ABCABC} \ldots$ arrangements characterize the diamond lattice, whereas when they are stacked as $\mathrm{ABAB} \ldots$ arrangements they give rise to the lonsdaleite lattice. The carbon skeleton of the hydrocarbon tetracyclo[5.3.1.1. $\left.1^{2,6} 0^{4,9}\right]$ dodecane, $\mathrm{C}_{12} \mathrm{H}_{18}$ (with the partition $\left(\mathrm{CH}_{2}\right)_{6}(\mathrm{CH})_{6}$, named iceane because water crystallizes forming such H-bonded aggregates) constitutes one of the units in the lonsdaleite lattice; other units have 8 carbon atoms. In iceane there are two disconnected chair-shaped and three boat-shaped hexagons. Nanotubes formed from 'hydrogen-depleted iceane units sharing chair-shaped hexagonal faces', shown in Figure 20 with ten such units, have only tertiary carbon atoms and therefore appear to be simpler than those involving adamantane units shown earlier (see Figure 14).

As in previous cases, one can convert nanotubes into tori, and Figure 21 shows such a torus having 28 'hydrogen-depleted iceane units sharing chair-shaped hexagonal faces'.

\section{CONCLUSIONS}

Assemblies of adamantane units (polymantanes) sharing chair-shaped six-membered rings of carbon atoms are hydrocarbons with low steric strain, which being the
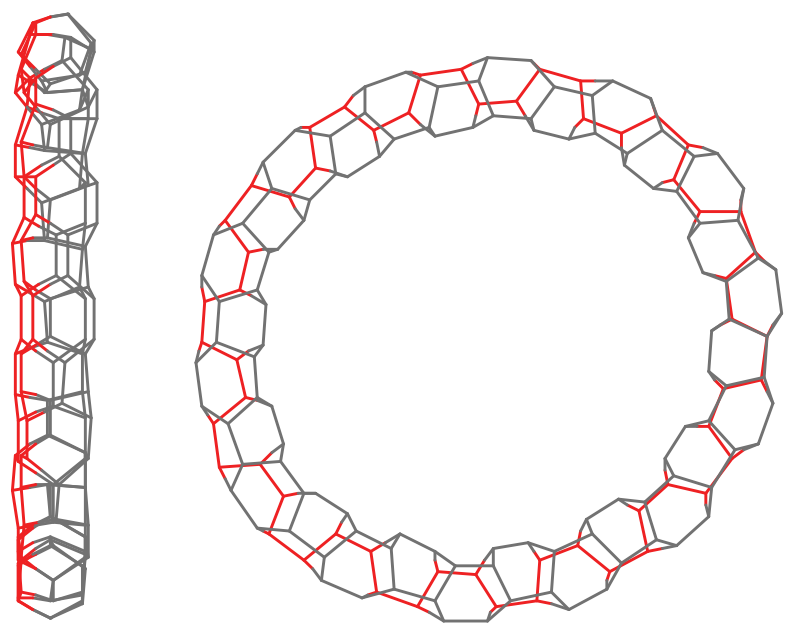

Figure 21. Toroidal structure obtained by joining the ends of a nanotube from the lonsdaleite lattice: views along the 'ring plane' and othogonal to the 'ring plane'. 
most stable among all their isomers have been thermodynamically favored and are found in petroleum. As discussed earlier without enetering into detail, ${ }^{17}$ adamantane units may also share single carbon atoms yielding spiromantanes, one or two $\mathrm{C}-\mathrm{C}$ bonds (oneedge- or two-edge-mantanes). All these possibilities have been and exemplified, paying special attention to macrocyclic assemblies of adamantane units.

What has the previous discussion to do with realworld chemistry, in addition to other, yet unknown oil constituents? A brief survey follows. Inside nanotubes of sufficiently large diameter (larger than $1 \mathrm{~nm}$ ), diamantane-4,9-dicarboxylic acid is converted into a diamond nanowire ([121212...]polymantane), a zigzag long hexagon-sharing polymantane. ${ }^{35}$ This is explained by the "capillary force" pulling the long molecules into the nanotube where the resulting huge pressure (above $0.4 \mathrm{GPa}$ ) causes decarboxylation and polymerization in a hydrogen-rich surrounding. Electron-beam irradiation dehydrogenates the enclosed polymantane into onion nanotubes. A more direct probe of producing diamonddoid nanothreads with local diamond-like structure by compressing benzene to $20 \mathrm{GPa}$ at room temperature. ${ }^{36}$

Reinhardt reported in 1962 the synthesis of the the simplest open-necklace-type biadamantane, ${ }^{37}$ and more recently Japanese authors prepared similar opennecklace-type tetramers of 1,3-adamantane derivatives. $^{38}$

The synthesis of tetracyclo[5.3.1.1 $\left.1^{2,6} 0^{4,9}\right]$ dodecane was announced in $1975^{39}$ with the proposed name "wurtzitane", but the name of iceane proposed one year later gained general acceptance. ${ }^{40}$ This is the building block of lonsdaleite, the hexagonal carbon allotrope, occurring in microcrystals formed in high-impact meterorites. $^{41,42}$

One-edge-[2]mantane was prepared by Schleyer and coworkers ${ }^{27,43}$ and was called [2]diadamantane, according to the number of shared vertices. Murray Jr. and coworkers achieved the regioselective functionalization of this compound at its tertiary $\mathrm{CH}$ groups. ${ }^{44}$ The same research group ${ }^{45}$ described the regioselective bromination at tertiary carbon atoms of [2] spiromantane (called [1]diadamantane according to Schlyer's proposal). ${ }^{27,43}$

\section{REFERENCES}

1. J. E. Dahl, S. G. Liu, and R. M. K. Carlson, Science 299 (2003) 96-99.

2. J. E. Dahl, J. M. Moldowan, K. E. Peters, G. E. Claypool, M. A. Rooney, G. E. Michael, G. E. Mello, and M. L. Kohnen, Nature 399 (1999) 54-57.

3. A. T. Balaban, D. J. Klein, J. E. Dahl, and R. M. K. Carlson, The Open Org. Chem. J. 1 (2007) 13-31.

4. (a) P. von R.Schleyer, My thirty years in hydrocarbon cages: From adamantane to dodecahedrane, in: Cage Hydrocarbons (G. A. Olah, editor), Wiley-Interscience, New York, 1990, pp.
1-38; (b) P. von R. Schleyer, J. Am. Chem. Soc. 79 (1957) 3292.

5. C. A. Cupas, P. von R. Schleyer, and D. J. Trecker, J. Am. Chem. Soc. 87 (1965) 917-918.

6. V. Z. Williams, Jr., P. von R. Schleyer, G. J. Gleicher, and L. B. Rodewald, J. Am. Chem. Soc. 88 (1966) 3862-3863.

7. (a) M. A. McKervey, Chem. Soc. Rev. 3 (1974) 479512; (b) M. A. McKervey, Tetrahedron 36 (1980) 974992; (c) M. A. McKervey and J. J. Rooney, in: Cage Hydrocarbons (G. A. Olah, editor), Wiley-Interscience, New York, 1990, pp. 39-64; (d) W. Burns, T. R. B. Mitchell, M. A. McKervey, J. J. Rooney, G. Ferguson, and P. Roberts, Chem. Comm. 21 (1976) 893-895.

8. P. R. Schreiner, A. A. Fokin, H. P. Reisenauer, B. A. Tkachenko, E. Vass; M. M. Olmstead, D. Bläser, R. Boese, J. E. P. Dahl, and R. M. K. Carlson, J. Am. Chem. Soc. 131 (2009) 11292-11293.

9. R. C. Fort, Jr. and P. von R Schleyer, Chem. Rev. 64 (1964) 277-300.

10. R. C. Fort, Jr., Adamantane. The Chemistry of Diamond Molecules, Marcel Dekker, New York, 1976.

11. (a) G. A. Mansoori, P. L. B. de Araujo, and E. S. de Araujo, Diamondoid Molecules. With Applications in Biomedicine, Materials Science, Nanotechnology, and Petroleum Science. World Scientific, New Jersey, 2012; (b) G. A. Mansoori, Diamondoid molecules, in: Advances in Chemical Physics 136 (2007) 207-258 (S. A. Rice, Ed.), Wiley, Hoboken..

12. A. T. Balaban, J. Chem. Inf. Model. 52 (2012) 2856-2869.

13. A. T. Balaban, J. Math. Chem. 51 (2013) 1043-1055:

14. H. Schwertfeger, A. A. Fokin, and P. R. Schreiner, Angew. Chem. Int. Ed. 47 (2008) 1022-1036.

15. A. T. Balaban and F. Harary, Tetrahedron 24 (1968) 2505-2516.

16. A. T. Balaban, Tetrahedron 25 (1969) 2949-2956.

17. A. T. Balaban and P. v. R. Schleyer, Tetrahedron 34 (1978) 3599-3609.

18. A. T. Balaban, Rev. Roum. Chim. 21 (1976) 1049-1071.

19. A. T. Balaban, MATCH, Commun. Math. Comput. Chem. 2 (1976) 51-61.

20. A. A. Fokin, B. A. Tkachenko, P. A. Gunchenko, D. V. Gusev, and P. R. Schreiner, Chem. Eur. J. 11 (2005) 7091-7101.

21. H. Schwertfeger, C. Würtele, H. Hausmann, J. E. P. Dahl, R. M. K. Carlson, A. A. Fokin, and P. R. Schreiner, Adv. Synth. Catal. 451 (2009) 1041-1054.

22. A. A. Fokin, P. A.; Gunchenko, A. A. Novikovsky, T. E. Shubina, B. V. Chernyaev, J. E. P. Dahl, R. M. K. Carlson; A. G. Yurchenko, and P. R. Schreiner, Eur. J. Org. Chem. 2009, 5153-5161.

23. A. A. Fokin, B. A. Tkachenko, N. A. Fokina,H. Hausmann, M. Serafin, J. E. P. Dahl, R. M. K. Carlson, and P. R. Schreiner, Chem. Eur. J. 15 (2009) 3851-3862.

24. A. A. Fokin, A. Merz, N. A. Fokina, H. Schwertfeger, S. L. Liu, J. E. P. Dahl, R. M. K. Carlson, and P. R. Schreiner, Synthesis 6 (2009) 909-912.

25. J. E. P. Dahl, J. M. Moldowan, Z. Wei, P. A. Lipton, P. Denisevich, R. Gat, S. Liu, P. R. Schreiner, and R. M. K. Carlson, Angew. Chem. Int. Ed. 49 (2010) 9881-9885

26. A. A. Fokin, T. S. Zhuk, A. E. Pashenko, P. O. Dral, P. A. Gunchenko, J. E. P. Dahl, R. M. K. Carlson, T. V. Koso, M. Serafin, and P. R. Schreiner, Org. Lett. 11 (2009) 3068-3071.

27. A. A. Fokin and P. R. Schreiner, Mol. Phys. 107 (2009) 823-830.

28. E. Boelema, J. Strating, and H. Wynberg, Tetrahedron Lett. 13 (1972) 1175-1177.

29. W. D. Graham and P. v. R.Schleyer, Tetrahedron Lett. 13 (1972) 1179-1180.

30. J. S. Lomas, C. Cordier, and S. Briand, S. J. Chem. Soc. Perkin Trans. 2 (1996) 865-870. 
31. J. S. Lomas, C. Cordier, S. Briand, and J. Vaissermann, J. Chem. Soc. Perkin Trans. 2 (1996) 871-876.

32. G. Rücker and C. Rücker, Chimia 44 (1990) 116-120.

33. A. T. Balaban and C. Rücker, Central Eur. J. Chem. 11 (2013) 1423-1430.

34. A. T. Balaban and C. Rücker, Arkivoc 2014 (2014) 346-361.

35. J. Zhang, Z. Zhu, Y. Feng, H. Ishiwata, Y. Miyata, R. Kitaura, J. E. P. Dahl, R. M. K. Carlson, N. A. Fokina, P. R. Schreiner, D. Tomanek, and H. Shinohara, Angew. Chem. Int. Ed. 52 (2013) 3717-3721.

36. T. C. Fitzgibbons, M. Guthrie, E. Xu, V. H. Crespi, S. K. Davidowski, G. D. Cody, N. Alem, and J. V. Badding, Nature Mat. 13 (2014), doi: 10.1038/nmat4088.

37. H. F. Reinhardt, J. Org. Chem. 27 (1962) 3258-3261.

38. T. Ishizone, H. Tajima, S. Matsuoka, and S. Nakahama, Tetrahe- dron Lett. 42 (2001) 8645-8647.

39. H. Tobler, R. O. Klaus, and C. Ganter, Helv. Chim. Acta 58 (1975) 1455-1464.

40. D. P. G. Hamon and G. F. Taylor, Austral. J. Chem. 29 (1976) 17211734

41. C. Frondel and U. B. Marvin, Nature 214 (1967) 587-589.

42. F. P. Bundy and J. S. Kasper, J. Chem. Phys. 46 (1967) $3437-$ 3446

43. W. D. Graham, P. v. R. Schleyer, E. W. Hagaman, and E. Wenkert, J. Am. Chem. Soc. 93 (1973) 5785-5786.

44. J. J. Sosnowski and R. K. Murray, Jr., J. Org. Chem. 49 (1984) $4471-4475$

45. J. J. Sosnowski, A. L. Rheingold, and R. K. Murray, Jr., J. Org. Chem. 50 (1985) 3788-3791. 